\title{
Convection-driven planetary dynamos
}

\author{
Ulrich R. Christensen ${ }^{1}$, Julien Aubert ${ }^{2}$ \\ and Peter Olson ${ }^{3}$ \\ ${ }^{1}$ Max-Planck-Institut for Solar System Research, 37191 Katlenburg-Lindau, Germany \\ email: Christensen@mps.mpg.de \\ ${ }^{2}$ Institut de Physique du Globe de Paris, Paris, France \\ email: aubert@ipgp.jussieu.fr \\ ${ }^{3}$ Department of Earth \& Planetary Sciences, Johns Hopkins University, Baltimore MD, USA \\ email: olson@jhu.edu
}

\begin{abstract}
Numerical simulations of convection-driven dynamos in rotating spherical shells are employed to better understand the observed strength and geometry of planetary magnetic fields. The model computations cannot be performed for realistic values of several of the control parameters. By varying parameters within the accessible range, it is possible to derive scaling laws for the magnetic field strength and the flow velocity in the dynamo region and for the dipole moment. Our scaling laws suggest that, even though diffusivities are far too large in the models, diffusive processes do not play an important role, just as in planetary cores. Extrapolating the scaling laws to planetary values of the control parameters leads to reasonable predictions for the field strength in the dynamo region and fits the observed dipole moments decently, in particular in the cases of Earth and Jupiter. For Mercury, which does not fit well when applying the scaling laws in a straightforward way, a model is proposed in which the upper part of the fluid core is stably stratified and the dynamo operates only in its deep regions. The time-varying dynamo field must diffuse through the stable region and is attenuated by the skin effect. The model explains why Mercury has a very weak but probably dipole-dominated magnetic field.
\end{abstract}

Keywords. Magnetic fields, convection, MHD, planets and satellites: general

\section{Introduction}

Most planets and a few satellites in the solar system have a global magnetic field of internal origin or they once had such a field. The magnetic field strengths at planetary surfaces cover a wide range, from $\approx 400 \mathrm{nT}$ for Mercury to $\approx 500,000 \mathrm{nT}$ in the case of Jupiter. In most cases the field is dominated by the axial dipole component, but the magnetic fields of Uranus and Neptune have strongly tilted dipoles and large multipole contributions. All these fields are thought to be generated by a dynamo process that is maintained by thermal and compositional convection in the fluid and electrically conducting cores of the planets (Stevenson 2003). The challenge for a unifying theory of planetary magnetism is to explain the diversity of magnetic field strength and structure. Scaling laws that relate the magnetic field strength to basic planetary parameters, such as rotation rate, core size and energy flux, have been proposed in the past based mainly on heuristic arguments (e.g. Stevenson 1979, Starchenko \& Jones 2002) and their merits still need to be assessed.

Numerical dynamos driven by convection in rotating fluid shells often produce magnetic fields similar to the geomagnetic field, with a strong axial dipole component, secular variation, and occasional polarity reversals (Kono \& Roberts 2002). However, problems remain in applying numerical model results directly, because the dynamo models are far removed in parameter space from the planets. Specifically, numerical dynamos rotate 
too slowly, are less turbulent and have far too large viscosity compared to planetary conditions. In terms of the non-dimensional parameters that govern convection-driven rotating dynamos, this means that the Rayleigh number is too small and the Ekman number and magnetic Prandtl number are too large (Glatzmaier 2002). Direct numerical simulation with realistic values would require much higher temporal and spatial resolution than what is feasible in the foreseeable future.

In this paper we review scaling laws derived recently from numerical dynamos within the part of parameter space now accessible to computation. First we consider how properties inside the dynamo region, such as the characteristic flow velocity, the magnetic field strength, and the efficiency of heat transport, depend on the control parameters. We then consider scaling laws for the dipole moment as the basic observable dynamo property.

While our scaling laws explain the observed magnetic field properties of most planets decently well, the weakness of Mercury's magnetic field poses a problem. Numerical simulations of Mercury's dynamo assuming either a very small or a very large solid inner core (Heimpel et al. 2005, Stanley et al. 2005, Takahashi \& Matsushima 2006) exhibit relatively low field strengths. However, their dipole moments are still too large by a factor of ten, or the model field at the planet's surface is dominated by higher multipole components, which does not agree well with the available (limited) observations by Mariner 10. We describe a recently proposed model in which the upper part of Mercury's core is stably stratified and the dynamo operates only at depth.

\section{Scaling internal dynamo properties}

In Christensen \& Aubert (2006) we studied numerically an extensive set of dynamo models in a rotating spherical shell with the geometry of Earth's core, covering a wide range of control parameters. Convection is driven from below and rigid boundaries are assumed. The Ekman number $E=\nu /\left(\Omega D^{2}\right)$ varies between $3 \times 10^{-6}$ and $3 \times 10^{-4}$, the magnetic Prandtl number $P m=\nu / \lambda$ between 0.06 and 10, the Prandtl number $\operatorname{Pr}=\nu / \kappa$ between 0.1 and 10, and the (modified) Rayleigh number $R a^{*}=\alpha g \Delta T /\left(\Omega^{2} D\right)$ is up to 50 times critical. Here $\Omega$ is the rotation rate, $\nu$ kinematic viscosity, $D$ shell thickness, $\lambda$ magnetic diffusivity, $\kappa$ thermal diffusivity, $\alpha$ thermal expansion coefficient, $g$ gravity, and $\Delta T$ the imposed temperature contrast between the rigid boundaries. We find two distinct classes of solutions with strong and weak dipole contributions to the overall magnetic field, respectively. The transition from dipolar to non-dipolar dynamos occurs when a local (scale-dependent) Rossby number, $R o_{\ell}=U /(\Omega \ell)$, exceeds a value of $\approx 0.12$, independent of the values of $E, \operatorname{Pr}$ and $\operatorname{Pm}(U$ is the rms-velocity and $\ell$ a characteristic length scale of the flow). $R o_{\ell}$ is a measure for the ratio of inertial forces to the Coriolis force. Hence dipolar dynamos break down when inertia starts to play a significant role in the force balance (see also Sreenivasan \& Jones 2006).

We find that in the dipolar regime the minimum magnetic Reynolds number $R m=$ $U D / \lambda$ for self-sustained dynamos is independent of the magnetic Prandtl number Pm in the range 40 - 50. However, dynamos at low Pm exist only at sufficiently low Ekman number $E$. The lowest magnetic Prandtl number at which a self-sustained dipolar dynamo is found varies as $P m \sim E^{3 / 4}$. At low $P m$ the hydrodynamic Reynolds number must be large to exceed the critical value of $R \mathrm{~m}$. The associated inertial effects have an adverse influence on the dynamo and a low Ekman number is required to balance them by strong rotational forces.

In Christensen \& Aubert (2006) we concentrated on the dipolar regime in an attempt to establish scaling laws that fit the numerical results. Assuming that diffusive effects do not 


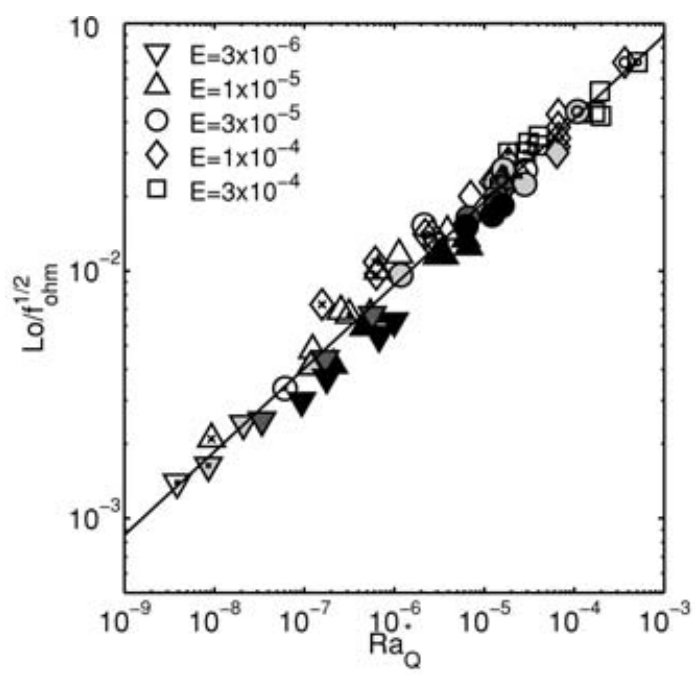

Figure 1. Lorentz number with dissipation correction factor versus modified Rayleigh number. The shape of the symbol indicates the value of the Ekman number and the shading indicates magnetic Prandtl number, where dark means $P m<1$. Small crosses in the main symbol mean $\operatorname{Pr}>1$ and circles $\operatorname{Pr}<1$. The slope of the correlation line is 0.34 .

play a primary role, we introduced non-dimensional parameters that are independent of any diffusivity (including kinematic viscosity). We take a modified Rayleigh number based on the advected heat flux $Q$ as the primary control parameter, $R a_{Q}^{*}=\gamma \alpha g Q /\left(\rho c_{p} \Omega^{3} D^{4}\right)$, which had been proposed earlier in the context of non-magnetic rotating convection (Christensen 2002). Here $\gamma$ is a numerical geometry factor, $\rho$ is density, and $c_{p}$ is heat capacity. The combination $F=\alpha Q / c_{p}$ is a buoyancy (or mass anomaly) flux and can be replaced by a buoyancy flux of arbitrary (e.g. compositional) origin. $R a_{Q}^{*}$ is equivalent to the non-dimensional power driving the flow. Characteristic properties of the solution are described by the global Rossby number $R_{o}=U /(\Omega D)$ for the flow velocity, the Lorentz number $L o=B /\left([\mu \rho]^{1 / 2} \Omega D\right)$ for the mean magnetic field strength $B$, and a modified Nusselt number $N u^{*} \sim Q /\left(\Omega \Delta T D^{3}\right)$ for the heat transport efficiency. To first approximation, all the dynamo results can be collapsed into simple power-law dependencies on the modified Rayleigh number:

$$
\begin{gathered}
R o=0.85 R a_{Q}^{* 0.41} \\
L o=0.92 f_{\text {ohm }}^{1 / 2} R a_{Q}^{* 0.34} \\
N u^{*}=0.076 R a_{Q}^{* 0.53}
\end{gathered}
$$

The scaling law for the Lorentz number involves a correction factor depending on $f_{\text {ohm }}$, the fractional contribution of ohmic dissipation to the total dissipation. Figure 1 shows the fit for the Lorentz number. In the empirical fits, the residual dependencies on the parameters related to diffusion $(E, P r, P m)$ are weak. The Ekman number and hydrodynamic Prandtl number seem to have no effect, but an influence of the magnetic Prandtl number, with a power law exponent of order 0.1, may exist. A similar weak dependency on $P m$ has been found before in a scaling law for the ohmic dissipation time in numerical dynamo models by Christensen \& Tilgner (2004), but has been rejected because it did not agree well with the ohmic dissipation observed in the Karlsruhe dynamo experiment (Stieglitz 2001) where Pm is much smaller than in the models. 
The Elsasser number $\Lambda=B^{2} /(\rho \mu \lambda \Omega)$ is the conventional measure for the ratio of Lorentz force to Coriolis force, and it is often assumed that the magnetic field in a planetary dynamo saturates at a value that makes $\Lambda$ to be of the order one (Stevenson 2003). However, in our numerical models $\Lambda$ is found to vary widely, in the range 0.1 - 100. The scaling law for the Lorentz number rather suggests that the magnetic field strength is controlled by the available power and not necessarily by a force balance. In fact, the finding that the fit is improved by the introduction of the correction factor $f_{\text {ohm }}$ is in agreement with a power-controlled field strength. In Christensen \& Aubert (2006) we tried to assess the relative importance of the various forces by studying sources and sinks of enstrophy (vorticity squared). In general the contributions of the Coriolis and buoyancy terms to the enstrophy budget are of the same order, inertial and viscous terms make smaller and variable contributions, and the Lorentz force term is highly variable. We could give only a partial theoretical basis for the scaling laws. The missing piece is an explanation for the empirical 2/5-exponent in the law for the Rossby number.

The scaling law for the Rossby number can be used to deduce the Rayleigh number of the Earth's core. Using core flow velocity estimates obtained from geomagnetic secular variation, we obtain $R a_{Q}^{*}$ to be about $3 \times 10^{-13}$ and an associated integrated buoyancy flux of $3 \times 10^{4} \mathrm{~kg} / \mathrm{sec}$. When we assume that this represents predominantly the compositional flux of light element in the fluid core which is rejected when the inner core solidifies, we find a small growth rate of the inner core of the order of $0.1 \mathrm{~mm} / \mathrm{yr}$ and an inner core age of the order 3.5 Gyr.

Taking a power law exponent of $1 / 3$ in the scaling law for the Lorentz number and ignoring $f_{o h m}$ and the possible weak dependence on $P m$, a surprising implication is that the mean magnetic field strength inside the dynamo $B$ is independent of both the conductivity and the rotation rate. This is in contrast to previously suggested magnetic field scaling laws (Stevenson 1979, Starchenko \& Jones 2002). B is basically controlled by the buoyancy flux. With a buoyancy flux of $3 \times 10^{4} \mathrm{~kg} / \mathrm{sec}$ we obtain a magnetic field strength slightly above $1 \mathrm{mT}$ inside the core. This is lower than most previous estimates, but is not unreasonable. It is in agreement with a value of $0.4 \mathrm{mT}$ for the field component pointing away from the rotation that has been inferred from the possible observation of torsional oscillations in the core (Zatman \& Bloxham 1997).

Applying our scaling laws to other planetary dynamos, we find that the observed excess luminosity of Jupiter implies an internal field of $8 \mathrm{mT}$, in agreement with Jupiter's external field being ten times stronger than that of the Earth. The weakness of Mercury's field cannot be explained by a very low buoyancy flux in the core, because this would correspond to a subcritical magnetic Reynolds number. We will discuss the issue of Mercury further in the following sections.

\section{Scaling of dipole moment}

In an attempt to find a general scaling law for the dipole moment, we used in Olson \& Christensen (2006) a larger and less homogeneous set of numerical dynamo results from the literature (Christensen et al. 1999, Christensen \& Aubert 2006, Glatzmaier 2002, Kutzner \& Christensen 2002, Kutzner \& Christensen 2004, Wicht \& Olson 2004, Takahashi et al. 2005, Takahashi \& Matsushima 2005) that covers a variety of boundary conditions and modes of driving convection. We do not restrict our analysis to dynamos that have a magnetic field dominated by the axial dipole, but include also the nondipolar regime.

We follow the same path as above to define the most suitable non-dimensional parameters. The non-dimensional dipole moment is given by a Lorentz number $L o_{d i p}=$ 
$(2 \mu / \rho)^{1 / 2} M /\left(4 \pi r_{o}^{3} \Omega D\right)$, where $M$ is the dipole moment and $r_{o}$ the outer radius of the fluid shell. Again $R a_{Q}^{*}$ is taken as the primary control parameter. A unique power-law scaling the relates $L o_{\text {dip }}$ to $R a_{Q}^{*}$ does not exist, rather the dipolar regime must be distinguished from the nondipolar one. In the latter the contribution of the dipole to the field at $r_{o}$ is weaker than that of higher multipoles. We had identified the local Rossby number $R o_{\ell}$ as the key parameter for controlling the dynamo regime in terms of the magnetic field structure. In Olson \& Christensen (2006) we found an empirical fit that relates it to the four basic control parameters. In terms of the physical properties it can be expressed as:

$$
R o_{\ell} \sim \frac{F^{1 / 2} \lambda^{1 / 5}}{\Omega^{7 / 6} D^{1 / 3} \nu^{1 / 3} \kappa^{1 / 5}},
$$

where $F$ is the buoyancy flux. While the transition from dipolar to nondipolar dynamos is rather abrupt at $R o_{\ell} \approx 0.12$ in the case of bottom-heated convection, it occurs more gradually and at lower values of $R o_{\ell}$ for internally heated dynamos. Interestingly, numerical dynamos that are generally dipole-dominated but show occasional reversals and excursions have a local Rossby number close to the transitional value.

In the dipolar regime the non-dimensional dipole moment can be expressed as

$$
L_{\text {dip }}=a R a_{Q}^{* 1 / 3}
$$

where $a$ is in the range of $0.1-0.2$. The fit is decent but not as good as in the case of the Lorentz number describing the internal dynamo field. This can be explained with the more heterogeneous set of model conditions employed to obtain equation (3.2), but also by the fact that the dipole is only one field component that contributes in a variable degree to the total field. In the nondipolar regime the dipole moments scatter more strongly, but the value of $L o_{d i p} / R a_{Q}^{* 1 / 3}$ is at least a factor of ten smaller than for dipole-dominated dynamos.

In Olson \& Christensen (2006) we apply our results to the various planets of the solar system. In most cases the uncertainties about the internal properties, in particular the buoyancy flux, are rather large and a kind of educated guess has to be made. Nonetheless, the dipole moments for Earth, Jupiter, and Saturn, where the constraints on internal properties can be considered to be fair or good, are in the predicted range within a factor of two. While Jupiter and Saturn are clearly in the dipolar regime according to our scaling, Earth is close to the transition point to the nondipolar regime. We suggest that occasional short lapses into the nondipolar structure may be the reason for geomagnetic excursions and reversals. Applying equation (3.1) to Mercury predicts it to be far on the nondipolar side, mainly because of the slow rotation. While this might explain the weakness of Mercury's observed field, it does not agree well with the relatively strong and perhaps dominant dipole contribution (Ness 1979). Special conditions may apply to Mercury's dynamo, which we discuss in the next section.

\section{A dynamo model for Mercury}

According to thermal evolution models (e.g. Hauck et al. 2004), the heat flow at Mercury's core-mantle boundary is expected to be a few $\mathrm{mWm}^{-2}$. This is less than the heat that can be conducted along an adiabatic temperature gradient in the core and therefore the outer core should be thermally stable. The evolution models also predict the existence of a solid inner core. The flux of light alloying elements from the boundary of a growing inner core could drive compositional convection. Because the unstable 


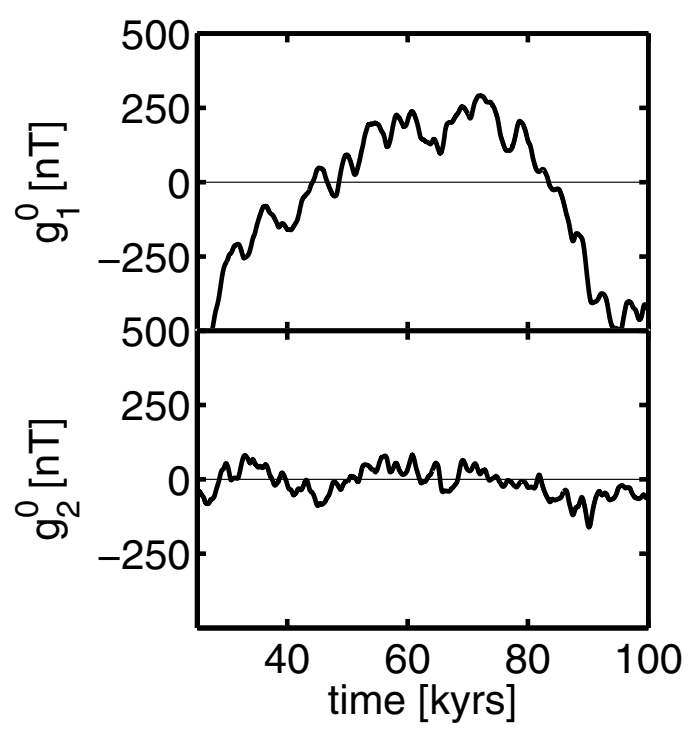

Figure 2. Gauss coefficients of the axial dipole field (upper panel) and axial quadrupole field as function of time at Mercury's surface. The best fit to the Mariner 10 data gives an absolute value of the dipole coefficient of $330 \mathrm{nT}$ (Ness 1979).

compositional gradient dominates near the inner core, whereas the stabilizing thermal gradient dominates at shallow depth, convection may be limited to a deep sublayer of the liquid core. Christensen (2006) has modelled convection and magnetic field generation in a partially stable fluid shell, with a positive buoyancy flux at the inner core boundary and a negative flux at the core-mantle boundary.

Guided by the scaling laws in section 2, the control parameters have been chosen such that the value of the magnetic Reynolds number in the dynamo region agrees with the one expected for plausible assumptions on the driving buoyancy flux. Furthermore, the parameters are in a range that leads to a nondipolar solution, in accord with our findings in section 3. We find that active convection is restricted to the unstable layer, roughly the lower $40 \%$ of the fluid shell in the models, although some toroidal flow occurs in the stable region. In the convective layer a strong field is generated, with an Elsasser number of order one. This spatial spectrum of the field has a broad maximum at spherical harmonic degrees in the range 7-10. The dipole and quadrupole contributions are weak in the dynamo region.

Christensen (2006) presented two model calculations. In case 1 the ratio of inner core radius to core radius is 0.35 and in case 2 it is 0.5 . The magnetic field at the planet's surface is dominated by the axial dipole, or temporarily by the axial quadrupole in case 1. The spatial power spectrum drops off rapidly for harmonic degrees $n$ larger than two. The average strength of the surface field is $20 \%$ of Mercury's observed surface field in case 1 and $200 \%$ in case 2 . In a third case, not reported before, the dipole moment (which varies with time) is of the same order as the observed moment of Mercury (Fig. 2). At all spatial scales the surface field is weaker than would be expected for a simple geometric decrease of amplitude from the dynamo region to the surface according to $r^{-(n+2)}$, particularly so at $n>3$. The only exception is the axial dipole in case 2 .

The main cause for the strong and selective reduction of the field strength outside the core is the skin effect. The dynamo field is time-dependent and must diffuse through the stable conducting layer in the outer parts of the core, which acts to first approximation as 
a filter that passes only low frequencies. Modes with higher $n$ tend to vary more rapidly with time, hence they are attenuated more strongly in comparison to those with low $n$. The axial dipole reverses on a 10,000 yr time scale in case 1 , twice in 100,000 yr in case 3 (Fig. 2), and not at all in case 2. Outside the core the axial dipole is moderately attenuated in case 1 , little attenuated in case 3 , and not at all damped in case 3 . In contrast, most non-dipole components vary on a thousand year or shorter time scale and are strongly damped. Therefore, the axial dipole can dominate the weak surface field although it is only a minor component of the dynamo field. The equatorial dipole also fluctuates rapidly, which makes the field outside the core much more axisymmetric than it is in the dynamo region. A phase shift accompanies the attenuation by the skin effect, so that variations in the dynamo field reach the core-mantle boundary with a time lag on the order of 3000 yrs.

\section{Discussion and Conclusions}

Numerical models of planetary dynamos suffer from the inability to adopt realistic values for several of the control parameters. Nonetheless, by varying the parameters in the accessible range, scalings law can be derived for the various dynamo properties. It is encouraging that these scaling laws are to first order independent of the viscosity and other diffusion constants, whose values cannot be matched to planetary values in the models. This suggests that present models are approaching already a dynamical regime where the diffusivities are unimportant, as it is expected to be the case in planetary cores. The results of applying the scaling laws to the geodynamo and other planetary dynamos are encouraging. A drawback is that so far the scalings laws are partly empirical. Challenges for the future are (1) to establish a more complete theoretical basis for the scaling laws, (2) further explore their range of validity, and (3) to clarify the possible influence of the magnetic Prandtl number. For the latter two points the comparison with future laboratory dynamo experiments will be very helpful.

A model where a dynamo operates below a stable layer in the outer parts of the fluid core can explain the properties of Mercury's magnetic field in terms of field strength and field geometry, as far as they are known from the limited Mariner 10 observations. The Messenger and Bepi Colombo missions will characterize the magnetic field much better. Some model predictions can be tested with new data, namely that the field has little energy at $n>3$, that it is predominantly axisymmetric, and that because of the frequency filtering of the skin effect no secular variation should observable on the time scale of decades.

\section{References}

Christensen, U.R. 2002, J. Fluid Mech. 470, 115

Christensen, U.R. 2006, Nature 444, 1056

Christensen, U., Olson, P. \& Glatzmaier, G.A. 1999, Geophys. J. Int. 138, 393

Christensen, U. R. \& Tilgner, A. 2004, Nature 439, 169

Christensen, U. R. \& Aubert, J. 2006, Geophys. J. Int. 166, 97

Glatzmaier, G.A. 2002, Annu. Rev. Earth Planet. Sci. 30, 237

Hauck, S.A., Dombard, A.J., Phillips, R.J. \& Solomon, S.C. 2004. Earth Planet. Sci. Lett. 222, 713

Heimpel, M.H., Aurnou, J.M., Al-Shamali, F.M. \& Gomez Perez, N. 2004. Earth Planet. Sci. Lett. 236, 542

Kono, M. \& Roberts, P.H. 2002, Rev. Geophys. 40, doi: 10.1029/2000RG000102

Kutzner, C. \& Christensen, U. R. 2002. Phys. Earth Planet. Int. 131, 29 
Kutzner, C. \& Christensen, U. R. 2004. Geophys. J. Int. 157, 1108

Ness, N.F. 1979. Phys. Earth Planet. Inter. 20, 209

Olson, P. \& Christensen, U. R. 2002. Geophys. J. Int. 151, 809

Olson, P. \& Christensen, U. R. 2006. Earth Planet. Sci. Lett. 250, 561

Starchenko, S.V. \& Jones, C.A. 2002, Icarus 157, 426

Stanley, S., Bloxham, J., Hutchinson, W.E. \& Zuber, M.T. 2005, Earth Planet. Sci. Lett. 234, 27

Sreenivasan, B. \& Jones, C.A. 2006, Geophys. J. Int. 164, 467

Stevenson, D.J. 1979, Geophys. Astrophys. Fluid Dyn. 12, 139

Stevenson, D.J. 2003, Earth Planet. Sci. Lett. 208, 1

Stieglitz, R. \& Müller, U. 2001, Phys. Fluids 13, 561

Takahashi, F. \& Matsushima, A. 2005, Phys. Fluids 17, 076601

Takahashi, F. \& Matsushima, M. 2006, Geophys. Res. Lett. 33, doi:1029/2006GL02579

Takahashi, F., Matsushima, M. \& Honkura, Y. 2005, Science 309, 459

Wicht, J. \& Olson, P. 2004, Geochem. Geophys. Geosyst. 5, doi:10.1029/2003GC000602

Zatman, S. \& Bloxham, J. 1997, Nature 388, 760

\section{Discussion}

G. RUEDIGER: In other geodynamo models I found a strong dependence of both geometry and amplitude of the induced magnetic field on the magnetic Prandtl number. In your presentation the magnetic Prandtl number did not play any role.

Christensen: Results for different values of the magnetic Prandtl number can be collapsed when control parameters and non-dimensional characteristic numbers are defined without refering to any of the diffusivities involved in the problem. The residual dependence on the magnetic Prandtl number is then weak.

J. ToOmRe: What do you believe happened to Mars and its magnetic dynamo ? Compositional convection not available?

Christensen: A likely scenario is that an early dynamo was driven by thermal convection, but that subsequently the heat flow from the core dropped below the heat that can be conducted along the adiabatic gradient, making the core thermally stable. The lack of plate tectonics on Mars implies that the mantle probably extracts less heat from the core than on Earth. Mars may have failed to nucleate a solid inner core, because of slower cooling and probably a high concentration of sulphur, which reduces the melting point. Hence compositional convection could be unavailable.

R. Rincon: Numerical results seem to agree well with observations in your case in spite of unrealistic viscosity/diffusivity astrophysical convection codes face the same situation. What can you suggest to check whether or not we get the right results for the right reason?

Christensen: You have to make an exhaustive study of parameter space and try to extract scaling laws. Then you may be able to extrapolate to computationally unreachable values of parameters. 